\title{
Downstaging Hepatocellular Carcinoma: A Systematic Review and Pooled Analysis
}

\author{
Neehar D. Parikh, ${ }^{1}$ Akbar K. Waljee, ${ }^{1}$ and Amit G. Singal ${ }^{2}$ \\ ${ }^{1}$ Division of Gastroenterology, University of Michigan Health System, University of Michigan, Ann Arbor, Ml; \\ ${ }^{2}$ VA Ann Arbor Health Services Research and Development Center of Clinical Management Research, \\ Ann Arbor, Ml; and ${ }^{3}$ Division of Digestive and Liver Diseases, University of Texas South Western Medical \\ Center, Dallas, $T X$
}

Downstaging can facilitate liver transplantation (LT) for patients outside of Milan criteria with hepatocellular carcinoma (HCC); however, the optimal protocol and downstaging outcomes are poorly defined. We aimed to characterize rates of successful downstaging to within Milan criteria and post-LT recurrence and survival among patients who underwent downstaging. We performed a systematic literature review using the MEDLINE and Embase databases from January 1996 through March 2015 and a search of national meeting abstracts from 2010 to 2014. Rates of downstaging success (defined as a decrease of tumor burden to within Milan) and post-LT recurrence with $95 \%$ confidence intervals (Cls) were calculated. Prespecified subgroup analyses were conducted by treatment modality, study design, and patient characteristics. Thirteen studies $(\mathrm{n}=950$ patients) evaluating downstaging success had a pooled success rate of $0.48(95 \% \mathrm{Cl}, 0.39-0.58 \%)$. In subgroup analyses, there was no significant difference comparing transarterial chemoembolization (TACE) versus transarterial radioembolization (TARE; $P=0.51$ ), but there were higher success rates in prospective versus retrospective studies ( 0.68 versus $0.44 ; P<0.001)$. The 12 studies $(n=320$ patients) evaluating post-LT HCC recurrence had a pooled recurrence rate of $0.16(95 \% \mathrm{Cl}, 0.11-0.23)$. There was no significant difference in recurrence rates between TACE and TARE $(P$ $=0.33$ ). Post-LT survival could not be aggregated because of heterogeneity in survival data reporting. Current data have heterogeneity in baseline tumor burden, waiting time, downstaging protocols, and treatment response assessments. There are also notable limitations including inconsistent reporting of inclusion criteria, downstaging protocols, and outcome assessment criteria. In conclusion, the success rate of downstaging HCC to within Milan criteria exceeds $40 \%$; however, posttransplant HCC recurrence rates are high at $16 \%$. Downstaging protocols for HCC should be systematically studied and optimized to minimize the risk of post-LT HCC recurrence. Liver Transpl 21:1142-1152, 2015. @ 2015 AASLD.

Received March 3, 2015; accepted May 3, 2015.

See Editorial on Page 1117
Liver transplantation (LT) is a lifesaving and curative treatment for patients with hepatocellular carcinoma (HCC). LT has been shown to provide the best long-

\footnotetext{
Additional supporting information may be found in the online version of this article.

Abbreviations: BCLC, Barcelona Clinic Liver Cancer; CI, confidence interval; EASL, European Association for the Study of the Liver; HCC, hepatocellular carcinoma; LT, liver transplantation; MOOSE, meta-analysis of observational studies in epidemiology; NOS, Newcastle-Ottawa scale; mRECIST, modified Response Evaluation Criteria in Solid Tumors; PRISMA, Preferred Reporting Items for Systematic Reviews and Meta-analysis; RFA, radiofrequency ablation; SBRT, stereotactic body radiation; TACE, transarterial chemoembolization; TALC, Transarterial Chemo-lipiodolization; TARE, transarterial radioembolization; UCSF, University of California-San Francisco; WHO, World Health Organization.

This work was conducted with support from the Agency for Health Research and Quality Center for Patient-Centered Outcomes Research (R24 HS022418). Akbar K. Waljee is funded by a Veterans Affairs Health Services Research and Development Career Development Program-2 Career Development Award 1IK2HX000775. The content is solely the responsibility of the authors and does not necessarily represent the official views of the University of Michigan or the Veterans Affairs Administration.

Potential conflict of interest: Nothing to report.

Address reprint requests to Neehar D. Parikh, M.D., M.S., Division of Gastroenterology, University of Michigan Health System, 3912 Taubman Center, 1500 East Medical Center Drive, Ann Arbor, MI 48109. Telephone: +1-734-936-8643; FAX: +1-734-936-7392; E-mail: ndparikh@med.umich.edu
}

DOI 10.1002/lt.24169

View this article online at wileyonlinelibrary.com.

LIVER TRANSPLANTATION.DOI 10.1002/It. Published on behalf of the American Association for the Study of Liver Diseases 
term survival for these patients, as it both cures the HCC and underlying liver disease. ${ }^{1}$ The number and proportion of LTs for HCC has increased in recent years, making it a leading indication for transplantation. ${ }^{2}$ The Milan criteria for LT ( 1 tumor less than 5 $\mathrm{cm}$, up to 3 tumors less than $3 \mathrm{~cm}$ in size) has been proposed, validated, and widely used as the eligibility criteria for consideration of LT in the treatment of HCC. ${ }^{3}$ Data from numerous cohorts of patients have shown that outcomes for LT in patients within Milan criteria are excellent with high survival rates and low recurrence rates. Overall, post-LT survival rates are comparable to patients transplanted without HCC. ${ }^{4,5}$

Unfortunately, only a minority of HCC patients present within Milan criteria. ${ }^{6}$ If patients present outside Milan criteria, LT is often not an option at many transplant centers and patients are left with no other curative options for their HCC. For selected patients, downstaging is attempted to bring tumors within Milan criteria by using liver-directed therapy. Options for conducting downstaging include radiofrequency ablation (RFA), transarterial chemoembolization (TACE), transarterial radioembolization (TARE), stereotactic body radiation (SBRT), or a combination of therapies. The benefits of downstaging include decreasing tumor burden and allowing time to identify those with less aggressive tumor biology.

There have been numerous reports of using downstaging to bring patients within transplant criteria; however, these data are largely restricted to small cohorts of patients and include disparate downstaging protocols. Therefore, the success rates and posttransplant outcomes after downstaging are largely unknown outside of single-center reports. We hypothesize that downstaging yields high success rates but is associated with high post-LT recurrence rates. To better characterize the efficacy of downstaging, we performed a meta-analysis of observational studies with individuals beyond Milan criteria who underwent downstaging with locoregional therapies. The aims of this meta-analysis were (1) to determine the success of downstaging $\mathrm{HCC}$ to bring patients within Milan criteria and (2) to characterize post-LT recurrence rates and survival of patients who were downstaged.

\section{PATIENTS AND METHODS}

\section{Literature Search}

Two study investigators (N.D.P. and A.G.S.) independently conducted a computer-assisted search of the MEDLINE and Embase databases to identify relevant published articles. We searched the databases from January 1, 1996 through March 1, 2015 with the following keyword combinations: (downstaging OR down staging OR downstage OR down stage) AND (hepatocellular carcinoma OR HCC OR liver cancer). Our search was limited to human studies published in English. Manual searches of references from relevant articles were performed to identify studies that were missed by our computer-assisted search. Additional manual searches of Digestive Diseases Week, Ameri- can Association for the Study of Liver Diseases, European Association for the Study of the Liver (EASL), American College of Gastroenterology, and the International Liver Cancer Association from 2010 to 2014 were performed.

\section{Study Selection}

The search for studies and study selection was conducted with meta-analysis of observational studies in epidemiology (MOOSE) and Preferred Reporting Items for Systematic Reviews and Meta-analysis (PRISMA) guidance. $^{7,8}$ Two investigators (N.D.P. and A.G.S.) reviewed all publication titles of citations identified by the search strategy. Potentially relevant studies were retrieved, and selection criteria were applied. The articles were independently checked for inclusion by 2 investigators (N.D.P. and A.G.S.), disagreements were resolved through discussion, and consensus was reached on all articles. Our inclusion criteria were (1) cohort studies (retrospective or prospective); (2) studies evaluating downstaging in patients with cirrhosis and HCC; (3) studies in which downstaging was performed using surgical resection, RFA, TACE, TARE, SBRT, or a combination of therapies; and (4) studies that reported rates of success for downstaging patients to within Milan criteria using imaging criteria and/or posttransplant outcomes (including recurrence rates and/or survival) among those who were downstaged to within Milan criteria. We excluded articles that (1) evaluated investigational procedures; (2) evaluated systemic chemotherapeutic agents; (3) used explant data for evaluation of downstaging success; (4) had incomplete data for primary outcomes of interest; (5) included less than 5 patients; and/or (6) used surgical resection as the only method for downstaging patients. Studies in which a minority of patients had surgical resection as a downstaging modality were included. For studies that reported on duplicate cohorts of patients, we used the most updated cohort or the publication that provided the most complete baseline and follow-up data. Additional exclusion criteria included non-English language and nonhuman data.

\section{Data Extraction}

Two investigators (N.D.P. and A.G.S.) independently extracted required information from eligible studies using standardized forms developed by the investigators. We collected data on study design (retrospective versus prospective), inclusion/exclusion criteria, modality of downstaging treatment, baseline liver function, pretreatment tumor burden, waiting time to LT, requirement for mandatory wait time before LT, posttreatment tumor burden, time to recurrence after LT, post-LT survival, and overall length of follow-up. Authors were contacted via e-mail for any missing information. Study quality was rated by 1 investigator (N.D.P.) using the modified Newcastle-Ottawa scale (NOS). ${ }^{9}$ This instrument rates observational studies on a 


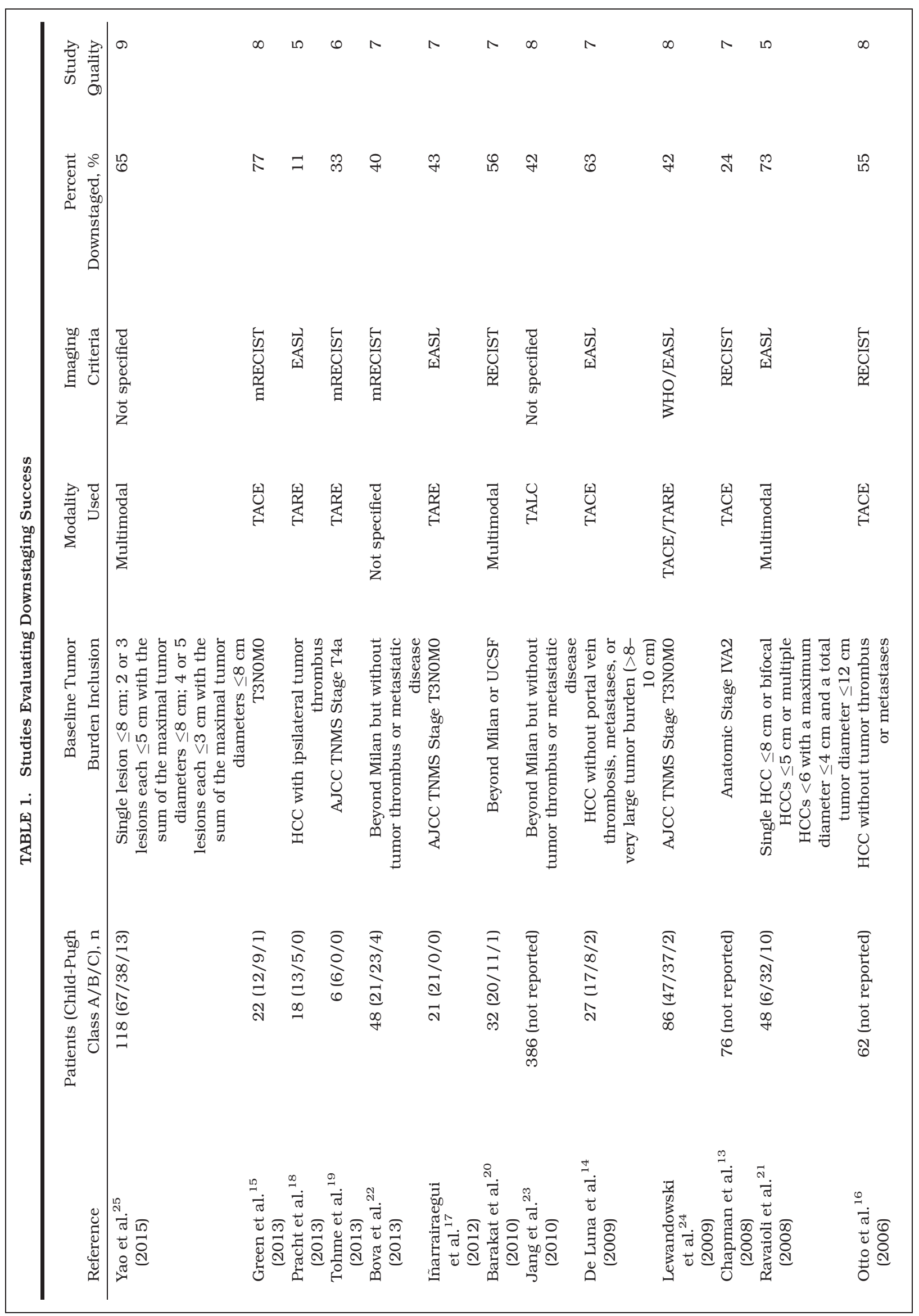




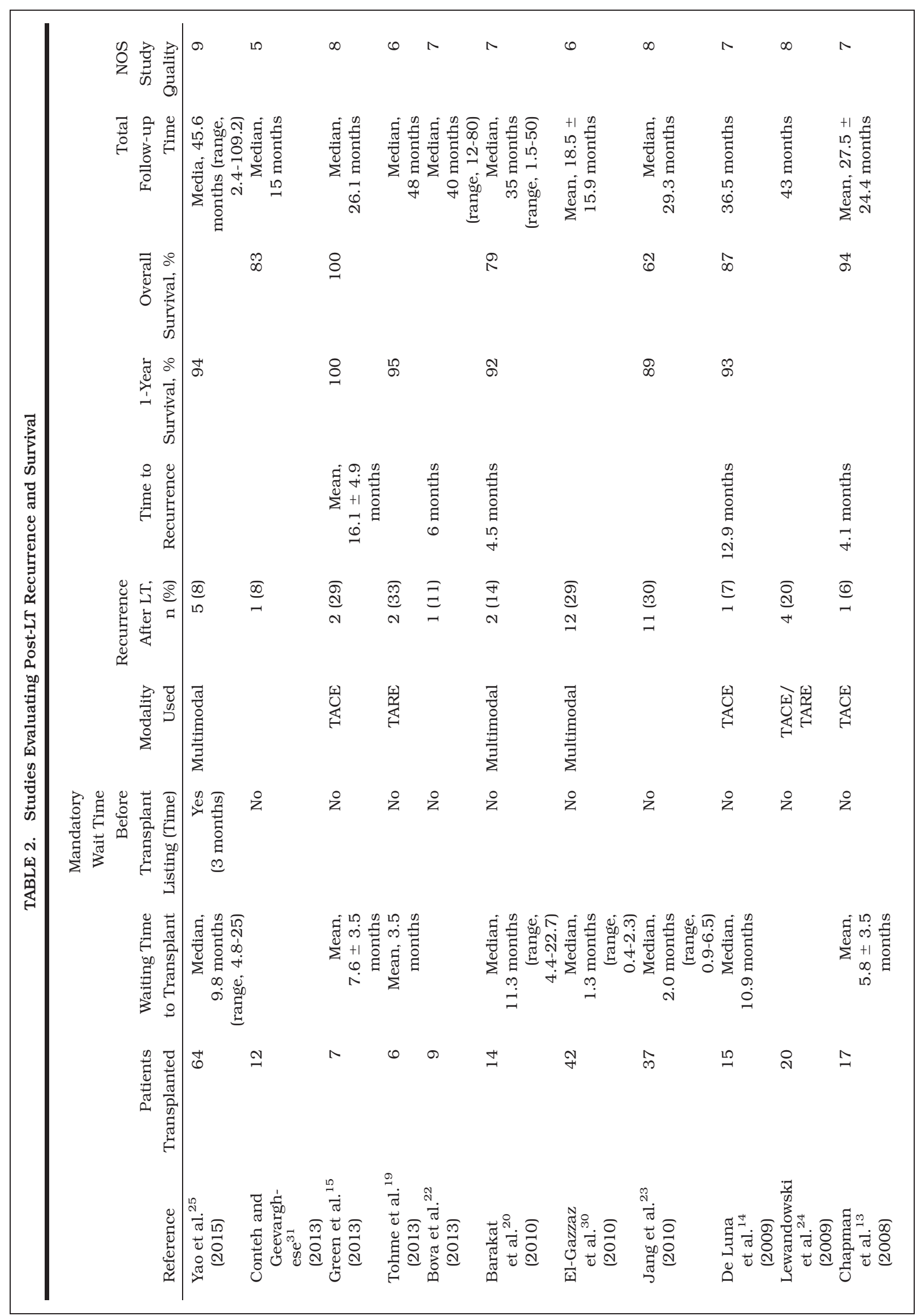




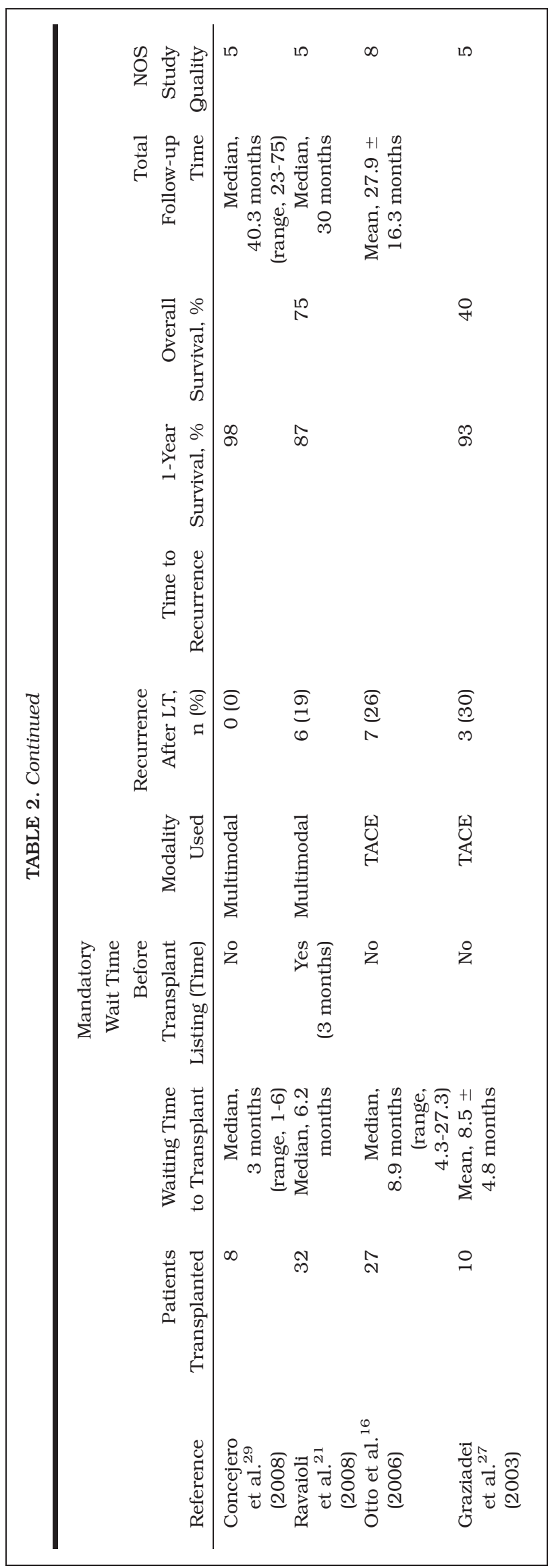

9-point scale on the basis of appropriateness of study sample, comparability of study groups, and adequacy of assessing exposure and outcomes. Studies with a score greater than 7 were considered high quality.

\section{Clinical Endpoints and Statistical Analysis}

The primary outcomes of interest were (1) success rate of downstaging to within Milan criteria and (2) HCC recurrence rates after LT. We also characterized post-LT survival but did not perform a pooled analysis given heterogeneity in the reporting of survival data. For both primary meta-analysis outcomes, we transformed proportions into a quantity using the Freeman-Tukey variant of the arcsine square roottransformed proportion. ${ }^{10}$ Pooled proportions for each outcome were calculated as a back-transform of the weighted mean of the transformed proportions using a random effects model. Heterogeneity was assessed using the $\mathrm{I}^{2}$ test and subset analyses. ${ }^{11}$ Subset analyses were planned for the following predefined subsets of studies: (1) TACE versus TARE; (2) prospective versus retrospective study design; (3) proportion of Child-Pugh class A patients; and (4) study quality. We included liver function as an a priori subset analysis given patients with preserved liver function may tolerate more aggressive and/or more cycles of downstaging treatment than patients with marginal liver function. We initially planned to do subset analyses based on baseline tumor burden, but this was not deemed possible given insufficient patient-level data. Publication bias was assessed by visual inspection of a funnel plot. ${ }^{12}$ All analyses were conducted in Stata 13.1 (StataCorp, College Station, TX).

\section{RESULTS}

\section{Literature Search}

The computer-aided search yielded 139 original articles, and the abstract of each article was reviewed. After reviewing the abstracts, 47 studies were independently reviewed by both investigators. After exclusions were applied, a total of 15 published studies were included in the final analysis. A review of the meeting abstract proceedings revealed 2 additional relevant studies for inclusion (Fig. 1). There were 13 studies with 950 unique patients which described the success of downstaging patients to within Milan criteria $^{13-25}$ (Table 1) and 15 studies with 320 unique patients which described posttransplant recurrence rates among patients who were downstaged (Table 2). ${ }^{13-16,19-30}$ Funnel-plot analysis showed similar effects among small and large studies for both outcomes with no evidence of publication bias (Supporting Figs. 1 and 2).

\section{Study Characteristics}

The majority of patients in the included trials had Child-Pugh class A disease (54\%), whereas patients with Child-Pugh class B (36\%) and Child-Pugh class 


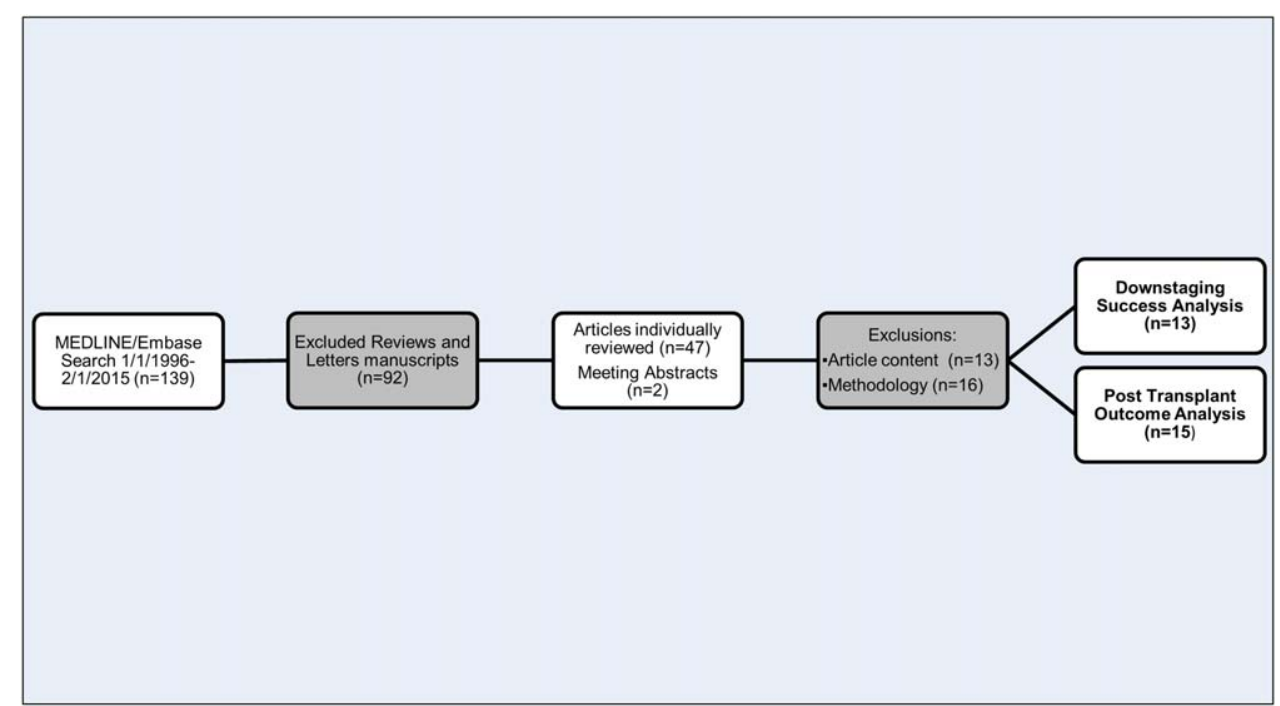

Figure 1. Search strategy.

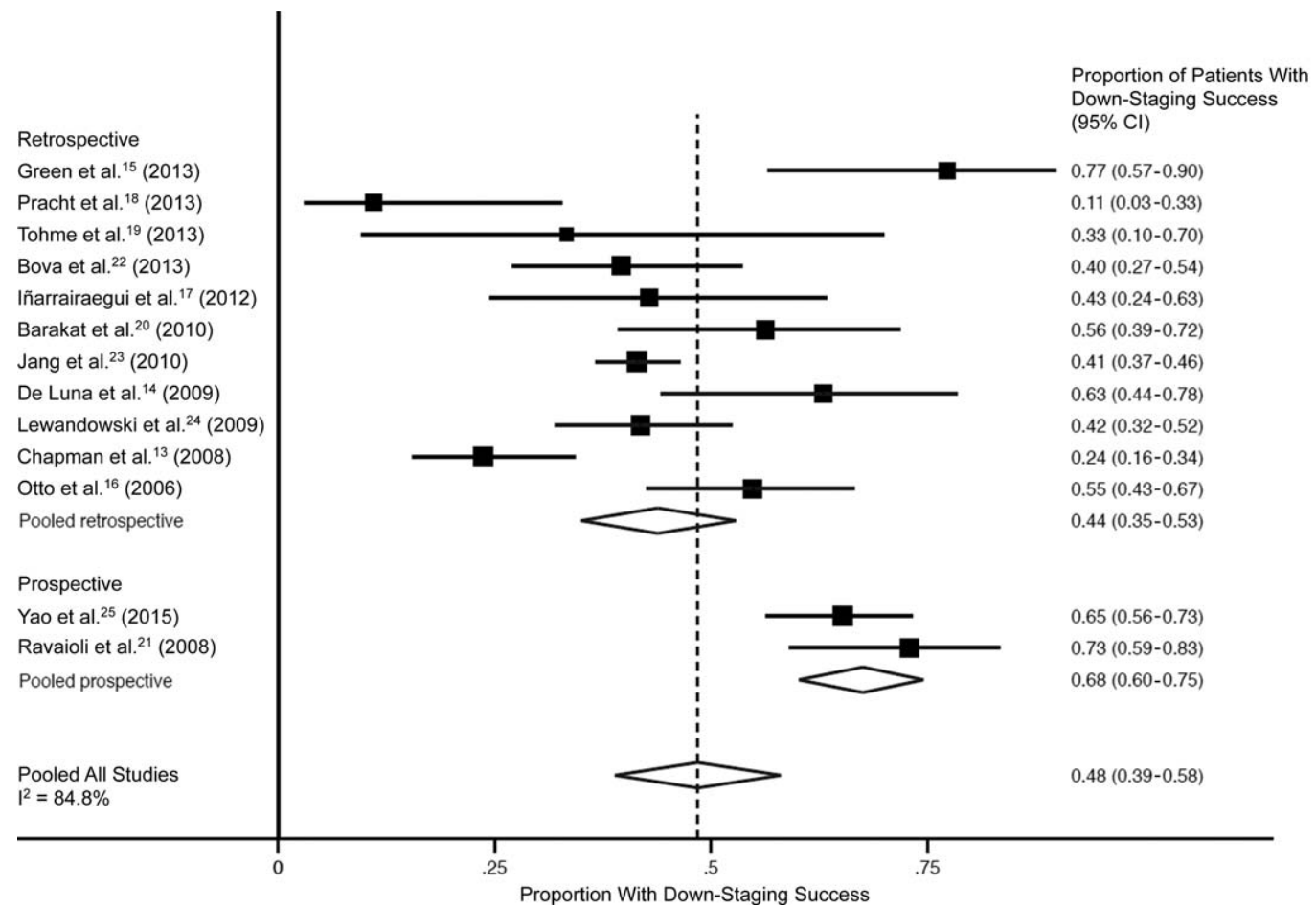

Figure 2. Pooled downstaging success stratified by prospective versus retrospective study design.

C $(8 \%)$ cirrhosis were less common. Initial tumor burden varied among studies (Table 1); however, only 2 studies included patients with tumor thrombus. ${ }^{13,18}$ Five studies ( $\mathrm{n}=230$ patients) used TACE as the primary downstaging modality, and 4 studies ( $\mathrm{n}=88$ patients) used TARE. Three studies ( $\mathrm{n}=198$ patients) described downstaging success in patients undergoing multimodal therapy. A minority of patients in multimodal studies ( $\mathrm{n}=15,8 \%$ ) underwent surgical resection as a downstaging modality. The number of treatment sessions and interval between treatment were not well described in the included studies. The imaging criteria used to define downstaging success also varied substantially, with EASL criteria being used in 4 studies, modified Response Evaluation Criteria in Solid Tumors (mRECIST) in 3 studies, RECIST in 3 studies, both EASL and World Health Organization (WHO) criteria in 1 study, and unspecified criteria in 2 studies.

After downstaging, 87 patients who underwent TACE and 15 patients who underwent TARE underwent LT. A total of 118 patients underwent LT after a multimodal downstaging approach. The waiting time between initiation of downstaging and LT as well as follow-up time after LT were inconsistently reported (Table 2). 


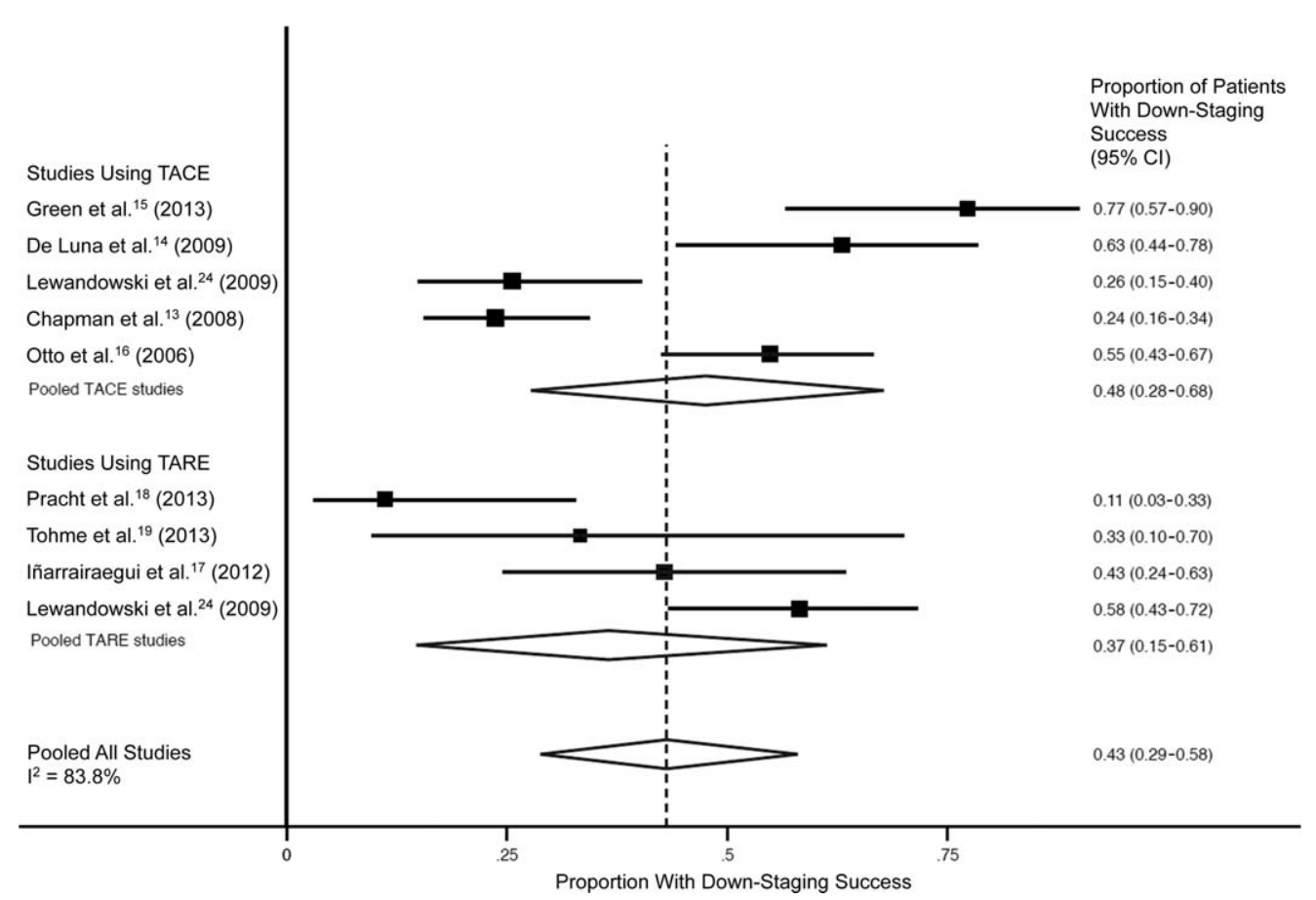

Figure 3. Pooled downstaging success stratified by treatment modality (TACE versus TARE).

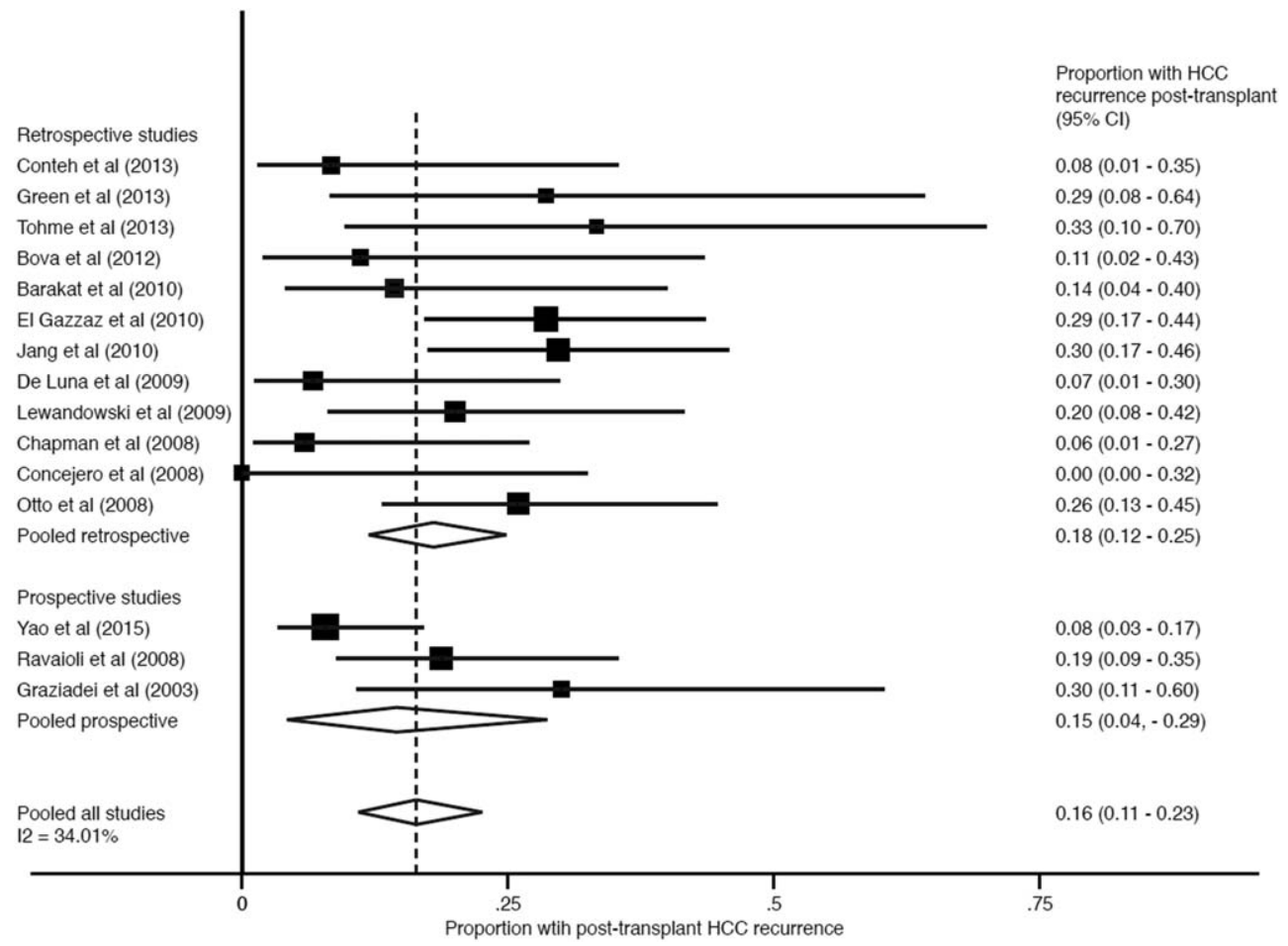

Figure 4. Pooled post-LT HCC recurrence stratified by prospective versus retrospective study design.

The quality of the included studies was primarily limited by small sample sizes, lack of comparator groups, poorly defined inclusion criteria, and/or inconsistent outcome assessment. The NOS quality scores are included in Table 2.

\section{Downstaging Success}

There was heterogeneity in downstaging success rate among included studies $\left(\mathrm{I}^{2}=84.8 \%\right)$, with an aggregate success rate of $0.48 \%$ (95\% confidence interval 


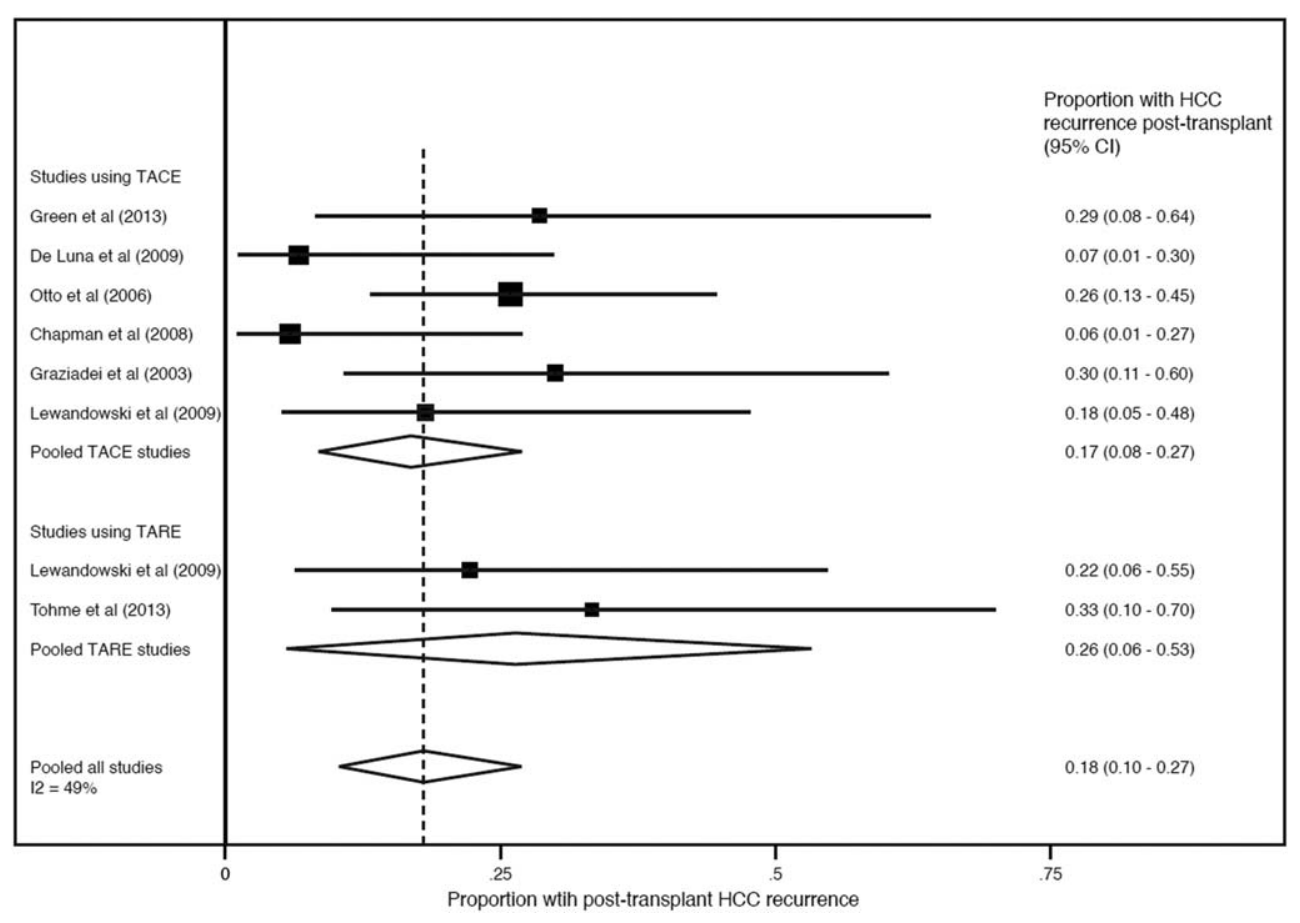

Figure 5. Pooled post-LT HCC recurrence stratified by treatment modality (TACE versus TARE).

[CI], 0.39\%-0.58\%). Studies that included patients with tumor thrombus had the lowest success rates; when these studies were excluded, the pooled success rate was $0.54 \%$ (95\% CI, 0.45\%-0.63\%). Studies with prospectively designed protocols for downstaging also yielded a significantly higher success rate compared to retrospective studies $(0.68 \%$ versus $0.44 \% P<$ 0.001 ; Fig. 2). There was no significant difference in the success rate of TACE and TARE for downstaging $(0.48 \%$ versus $0.37 \% ; P=0.51$; Fig. 3$)$; however, the highest downstaging success rates were reported in cohorts undergoing multimodal therapy for downstaging. The 3 studies using multimodal locoregional therapy ( $\mathrm{n}=198$ patients) had a significantly higher pooled downstaging success rate when compared to the success with TACE/TARE $(0.66 ; 95 \% \mathrm{CI}$, 0.58-0.73; $P<0.001) .{ }^{20,21,25}$ Contrary to our original hypothesis, studies including higher proportions of Child-Pugh A patients $(>60 \%)$ had lower rates of downstaging success than studies with less ChildPugh A patients (0.42 versus $0.58 ; P=0.019$ ); however, this difference was not statistically significant. There was no significant difference in downstaging success when studies were stratified into high- and low-quality studies (55\% versus $0.43 ; P=0.28$ ).

\section{Post-LT Recurrence}

In total, 58 (0.16; 95\% CI, 0.11-0.23) patients had HCC recurrence after LT; although studies had lower heterogeneity in terms of recurrence rates $\mathrm{I}^{2}=$ $33.5 \%$ ), there were substantial variations in the timeto-recurrence (Table 2). Similarly, wait times before LT were heterogeneous and inconsistently reported
(Table 2). In a sensitivity analysis excluding studies that allowed for tumor thrombus, the pooled post-LT recurrence rate was 0.16 (95\% CI, 0.11-0.23). There was no significant difference in recurrence rates between retrospective and prospective studies $(0.18$ versus 0.15 , respectively; $P=0.86$; Fig. 4 ). Two of the prospective studies mandated a 3 -month waiting time before listing for $\mathrm{LT},{ }^{21,25}$ whereas none of the other studies that were included had a similar requirement. The recurrence rate after LT did not differ significantly by downstaging modality $(0.17$ for TACE versus 0.26 for TARE; $P=0.40$; Fig. 5). Studies using multimodal downstaging techniques also had a similar pooled recurrence rate of 0.10 (95\% CI, 0.03-0.18; $\mathrm{I}^{2}, 19 \% ; P$ $=0.29$ ). Studies with a high proportion of Child-Pugh A patients had a similar recurrence rate versus those with a low proportion of Child-Pugh A patients $(13 \%$ versus $0.17 ; P=0.90$ ). Finally, recurrence rates did not differ by study quality, with high-quality studies having a recurrence rate of $0.20 \pm 0.11$ and lowquality studies having a recurrence rate of $0.15 \pm$ $0.06(P=0.58)$.

\section{Post-LT Survival}

There was variability in reporting of survival data, with several studies reporting 1-year and 3-year survival rates, others reporting mean or median survival, and some simply providing a Kaplan-Meier curve. Some studies reported survival as an intention-totreat analysis starting at time of downstaging, whereas others reported survival starting at time of LT for the subset of patients who were successfully downstaged. Most studies with post-LT survival data 
TABLE 3. Proposed Minimum Reporting Criteria for Downstaging Studies

Patient characteristics Demographics

Etiology of liver disease

Liver function (Child-Pugh class, presence of decompensation)

Center characteristics

LT volume

Median wait time for LT

Downstaging inclusion protocol

Tumor characteristics

Tumor burden (dominant tumor size, cumulative

tumor size)

BCLC tumor stage

Imaging modality and criteria used to measure tumor size (mRECIST, WHO, EASL)

Treatment details

Treatment modalities used (type, number)

Interval between initial downstaging and listing for

LT

Treatments received while awaiting LT

Wait time on the LT list

Time from initiation of downstaging to LT

LT details

Total length of follow-up post-LT

Immunosuppression protocol post-LT

Overall and recurrence-free graft survival

Overall and recurrence-free patient survival

reported 1-year survival rates exceeding 90\% (range, 87\%-100\%; Table 2); however, there was substantial variability in long-term survival rates. Although some studies had 4- or 5-year survival rates exceeding $90 \%,{ }^{13,29}$ others reported lower survival rates of approximately $70 \% .^{16,23,27}$ Heterogeneity in reporting of the data prevented pooling of survival data or meaningful subgroup analyses.

\section{DISCUSSION}

Downstaging $\mathrm{HCC}$ to within Milan criteria allows patients with advanced HCC to potentially have a curative treatment option. Overall, our pooled analysis shows that nearly half of the patients will achieve successful downstaging once initiated. However, downstaging is associated with higher posttransplant HCC recurrence rates compared to published data on patients who present within Milan. There appears to be little difference in success rates or posttransplant outcomes by downstaging modality; however, there are no randomized data comparing modalities.

Although the high posttransplant recurrence rates in this study are discouraging, it is worth noting that most studies to date have been retrospective in nature. We found significantly higher success rates in prospectively conducted studies compared to retrospective analyses. Similarly, post-LT recurrence rates were lower, although this difference did not reach statistical significance. These differences in outcomes were likely related to differences in study design including (1) stricter patient selection with more defined and limited tumor burden, (2) mandatory wait times before LT, and (3) higher consistency in quality of downstaging modalities. For example, the prospective study by Yao et al. $^{25}$ used strict inclusion criteria and a mandatory wait time before LT, resulting in the highest downstaging success rate and a lower recurrence rate than other included studies. A priori inclusion criteria for downstaging protocols appears to be a rational approach to maximizing the probability of success of achieving Milan criteria and reducing the risk of posttransplant recurrence. Other potential strategies to reduce posttransplant recurrence may include a mandatory waiting time and/or use of biomarker (eg, alpha-fetoprotein or des-gamma-carboxyprothrombin) cutoffs before consideration of transplantation in downstaging candidates. ${ }^{32}$ Only 2 studies included in our study, both prospective in nature, included a mandatory waiting period before listing for transplantation, emphasizing the importance of an institutional downstaging protocol. ${ }^{21,25}$

Although we found no difference in outcomes according to downstaging modality (TACE versus TARE), published data on TARE-related downstaging success and post-LT recurrence are limited. At least 1 of the TARE studies investigated downstaging exclusively in patients with tumor thrombus, which likely underestimates the potential effectiveness of TARE for downstaging in patients with liver-limited disease. ${ }^{18}$ Similarly, data on other emerging modalities, such as SBRT, are also sparse. Although a multimodal treatment approach yielded the best outcome in our analysis, all multimodal studies had a proportion $(8 \%)$ of patients who underwent surgical resection for downstaging. Patients eligible for surgery are typically without significant portal hypertension and therefore may have different clinical outcomes than other patients who receive locoregional therapies. Further studies evaluating and comparing different downstaging modalities are clearly needed.

One of the most notable findings of our systematic review is the substantial heterogeneity and limitations of data evaluating downstaging. First, there was substantial variation in baseline tumor burden-with some studies only including patients within University of California-San Francisco (UCSF) criteria (Barcelona Clinic Liver Cancer [BCLC] stage B), whereas others included patients with tumor thrombus (BCLC stage C). Second, there was variation in response assessment, with some studies using entire lesion size (RECIST or WHO) and others using viable tumor size (EASL or mRECIST). Third, a small number of studies included a mandatory waiting period, although most did not. Finally, we found important factors such as liver function, tumor burden at entry, and wait time before LT were not consistently reported. ${ }^{33}$

To improve the quality of literature evaluating HCC downstaging, we propose minimum reporting criteria for future studies (Table 3). Standardizing reporting of downstaging would allow for better understanding of 
data moving forward. Ideally, a multicenter wellpowered trial of downstaging protocols would be conducted to understand the optimal tumor burden, downstaging modality and protocol, and wait time before LT in order to achieve the best outcomes for downstaged patients. Alternatively, more stringent reporting of post-LT HCC recurrence rates could be mandated by the United Network for Organ Sharing in order to better characterize recurrence risk in this population of patients.

Our study has many strengths and limitations. As noted above, the included studies are heterogeneous with variable patient populations with regards to tumor burden, liver function, therapy received, and posttreatment response criteria. These differences make studies difficult to directly compare and thereby limit our ability to make strong conclusions about the superiority of one modality or protocol over another. Second, we did not have patient-level data to perform additional analyses on the basis of tumor burden or liver function. Given the small cohorts of single-center patient data, included studies may suffer from reporting bias. Our funnel plots showed no evidence of bias; however, this may reflect the large number of small studies included in this meta-analysis. ${ }^{34}$ In addition, non-English studies were excluded because of practical considerations, which may further bias the results. Nevertheless, our meta-analysis provides an important comprehensive summary of the downstaging success and posttransplant outcomes as reported in the literature. Furthermore, we highlight notable limitations of the current literature, which have informed our recommendations for minimum reporting criteria moving forward to improve our evidence base for downstaging.

In summary, we have shown that downstaging patients outside of Milan can be achieved in approximately half of all patients; however, post-LT recurrence is higher than what has been reported in patients who present within Milan. It is important to note that in well-designed studies with downstaging protocols, equivalent posttransplant results between downstaged patients and those who present within Milan criteria can be achieved. ${ }^{25}$ Although comparisons of downstaging modalities and protocols are difficult given the heterogeneity and limitations of current data, a protocolized approach with carefully patient selection and a standardized downstaging protocol is likely necessary to yield optimal outcomes. Large prospective studies, using standardized reporting criteria, are needed to compare downstaging modalities and protocols to optimize success and posttransplant survival in this cohort of patients.

\section{REFERENCES}

1. El-Serag HB, Siegel AB, Davila JA, Shaib YH, CaytonWoody M, McBride R, McGlynn KA. Treatment and outcomes of treating of hepatocellular carcinoma among Medicare recipients in the United States: a populationbased study. J Hepatol 2006;44:158-166.
2. Thuluvath PJ, Guidinger MK, Fung JJ, Johnson LB, Rayhill SC, Pelletier SJ. Liver transplantation in the United States, 1999-2008. Am J Transplant 2010;10(pt 2): 1003-1019.

3. Mazzaferro V, Regalia E, Doci R, Andreola S, Pulvirenti A, Bozzetti $F$, et al. Liver transplantation for the treatment of small hepatocellular carcinomas in patients with cirrhosis. N Engl J Med 1996;334:693-699.

4. Yao FY, Ferrell L, Bass NM, Bacchetti P, Ascher NL, Roberts JP. Liver transplantation for hepatocellular carcinoma: comparison of the proposed UCSF criteria with the Milan criteria and the Pittsburgh modified TNM criteria. Liver Transpl 2002;8:765-774.

5. Leung JY, Zhu AX, Gordon FD, Pratt DS, Mithoefer A, Garrigan K, et al. Liver transplantation outcomes for early-stage hepatocellular carcinoma: results of a multicenter study. Liver Transpl 2004;10:1343-1354.

6. Altekruse SF, Henley SJ, Cucinelli JE, McGlynn KA. Changing hepatocellular carcinoma incidence and liver cancer mortality rates in the United States. Am J Gastroenterol 2014;109:542-553.

7. Liberati A, Altman DG, Tetzlaff J, Mulrow C, Gøtzsche PC, Ioannidis JP, et al. The PRISMA statement for reporting systematic reviews and meta-analyses of studies that evaluate health care interventions: explanation and elaboration. PLoS Med 2009;6:e1000100.

8. Stroup DF, Berlin JA, Morton SC, Olkin I, Williamson GD, Rennie D, et al. Meta-analysis of observational studies in epidemiology: a proposal for reporting. Meta-analysis Of Observational Studies in Epidemiology (MOOSE) group. JAMA 2000;283:2008-2012.

9. Wells GA, Shea B, O'Connell D, Peterson J. The Newcastle-Ottawa Scale (NOS) for assessing the quality of nonrandomised studies in meta-analyses. 3rd Symposium on Systematic Reviews: Beyond the Basics; 2000.

10. Stuart A. Kendall's Advanced Theory of Statistics. 6th ed. Hoboken, NJ: Wiley; 2010.

11. Higgins JP, Thompson SG, Deeks JJ, Altman DG. Measuring inconsistency in meta-analyses. BMJ 2003;327: 557-560.

12. Egger M, Davey Smith G, Schneider M, Minder C. Bias in meta-analysis detected by a simple, graphical test. BMJ 1997;315:629-634.

13. Chapman WC, Majella Doyle MB, Stuart JE, Vachharajani N, Crippin JS, Anderson CD, et al. Outcomes of neoadjuvant transarterial chemoembolization to downstage hepatocellular carcinoma before liver transplantation. Ann Surg 2008;248:617-625.

14. De Luna W, Sze DY, Ahmed A, Ha BY, Ayoub W, Keeffe $\mathrm{EB}$, et al. Transarterial chemoinfusion for hepatocellular carcinoma as downstaging therapy and a bridge toward liver transplantation. Am J Transplant 2009;9:1158-1168.

15. Green TJ, Rochon PJ, Chang S, Ray CE Jr, Winston H, Ruef R, et al. Downstaging disease in patients with hepatocellular carcinoma outside of Milan criteria: strategies using drug-eluting bead chemoembolization. J Vasc Interv Radiol 2013;24:1613-1622.

16. Otto G, Herber S, Heise M, Lohse AW, Mönch C, Bittinger F, et al. Response to transarterial chemoembolization as a biological selection criterion for liver transplantation in hepatocellular carcinoma. Liver Transpl 2006; 12:1260-1267.

17. Iñarrairaegui M, Pardo F, Bilbao JI, Rotellar F, Benito A, D'Avola D, et al. Response to radioembolization with yttrium-90 resin microspheres may allow surgical treatment with curative intent and prolonged survival in previously unresectable hepatocellular carcinoma. Eur J Surg Oncol 2012;38:594-601.

18. Pracht M, Edeline J, Lenoir L, Latournerie M, Mesbah H, Audrain O, et al. Lobar hepatocellular carcinoma with 
ipsilateral portal vein tumor thrombosis treated with yttrium-90 glass microsphere radioembolization: preliminary results. Int J Hepatol 2013;2013:827649.

19. Tohme S, Sukato D, Chen HW, Amesur N, Zajko AB, Humar A, et al. Yttrium-90 radioembolization as a bridge to liver transplantation: a single-institution experience. J Vasc Interv Radiol 2013;24:1632-1638.

20. Barakat O, Wood RP, Ozaki CF, Ankoma-Sey V, Galati J, Skolkin M, et al. Morphological features of advanced hepatocellular carcinoma as a predictor of downstaging and liver transplantation: an intention-to-treat analysis. Liver Transpl 2010;16:289-299.

21. Ravaioli M, Grazi GL, Piscaglia F, Trevisani F, Cescon M, Ercolani G, et al. Liver transplantation for hepatocellular carcinoma: results of downstaging in patients initially outside the Milan selection criteria. Am J Transplant 2008;8:2547-2557.

22. BovaV,MiragliaR, MaruzzelliL, VizziniGB,LucaA. Predictive factors of downstaging of hepatocellular carcinoma beyond the Milan criteriatreated withintra-arterial therapies. CardiovascInterventRadiol2013;36:433-439.

23. Jang JW, You CR, Kim CW, Bae SH, Yoon SK, Yoo YK, et al. Benefit of downsizing hepatocellular carcinoma in a liver transplant population. Aliment Pharmacol Ther 2010;31:415-423.

24. Lewandowski RJ, Kulik LM, Riaz A, Senthilnathan S, Mulcahy MF, Ryu RK, et al. A comparative analysis of transarterial downstaging for hepatocellular carcinoma: chemoembolization versus radioembolization. Am J Transplant 2009;9:1920-1928.

25. Yao FY, Mehta N, Flemming J, Dodge J, Hameed B, Fix $\mathrm{O}$, et al. Downstaging of hepatocellular cancer before liver transplant: Long-term outcome compared to tumors within Milan criteria. Hepatology 2015;61:1968-1977.

26. Graziadei IW, Sandmueller H, Waldenberger P, Koenigsrainer A, Nachbaur K, Jaschke W, et al. Chemoembolization followed by liver transplantation for hepatocellu- lar carcinoma impedes tumor progression while on the waiting list and leads to excellent outcome. Liver Transpl 2003;9:557-563.

27. Shi XJ, Jin X, Wang MQ, Wei LX, Ye HY, Liang YR, et al. Outcomes of loco-regional therapy for downstaging of hepatocellular carcinoma prior to liver transplantation. Hepatobiliary Pancreat Dis Int 2011;10:143-150.

28. Concejero A, Chen CL, Wang CC, Wang SH, Lin CC, Liu YW, et al. Living donor liver transplantation for hepatocellular carcinoma: a single-center experience in Taiwan. Transplantation 2008;85:398-406.

29. El-Gazzaz G, Aucejo FN, Menon KN, Miller CM, Quintini C, Eghtesad B, et al. Outcome of pre-Transplant Locoregional Therapy to Downstage Hepatocellular Carcinoma (HCC). Abstracts of the American Association for the Study of Liver Diseases 61st Annual Meeting and Postgraduate Course. October 29-November 2, 2010; Boston, Massachusetts, USA. Hepatology 2010;52(suppl):320A-1291A.

30. Conteh L, Geevarghese S. Downstaging hepatocellular carcinoma: equivalent post-transplant outcomes among patients initially categorized beyond and within Milan Criteria. International Liver Cancer Association Annual Meeting, September 2013; Washington, DC:ILCA; 2013.

31. Toso C, Mentha G, Kneteman NM, Majno P. The place of downstaging for hepatocellular carcinoma. J Hepatol 2010;52:930-936.

32. Schlansky B, Chen Y, Scott DL, Austin D, Naugler WE. Waiting time predicts survival after liver transplantation for hepatocellular carcinoma: a cohort study using the United Network for Organ Sharing registry. Liver Transpl 2014;20:1045-1056.

33. Hunter JP, Saratzis A, Sutton AJ, Boucher RH, Sayers $\mathrm{RD}$, Bown MJ. In meta-analyses of proportion studies, funnel plots were found to be an inaccurate method of assessing publication bias. J Clin Epidemiol 2014;67: 897-903. 